\title{
THE BREAKTHROUGH IRONMAKING TECHNOLOGIES COMBINED WITH ENERGIRON, BLAST FURNACE AND SYNGAS*
}

Hiroshi Ichikawa ${ }^{1}$

Toshitaka Nakayama² Pablo E. Duarte 3

Alessandro Martinis ${ }^{4}$

\begin{abstract}
In 2013, Nippon Steel \& Sumikin Engineering Co., Ltd. (hereafter referred to as NSENGI), Tenova HYL and Danieli have entered into an agreement to combine ENERGION DR technology into blast furnace technology and/or with syngas technologies. This new alliance will allow the three companies to combine research and development activities with their respective expertise in ENERGIRON DR technology, Blast Furnace technology and Syngas (by-pro gas and coal gasification) technology, with the ultimate objective to develop breakthrough iron making technologies and create such EPC projects. This paper introduces the concepts and the features of our breakthrough technologies of combining of ENERGIRON DR technology, Blast Furnace technology and Syngas technology.

Keywords: DRI; Energiron; Blast Furnace; Syngas; Productivity; Coke rate; COG; Coal gasification.
\end{abstract}

Senior Manager, Plant \& Machinery Division, Nippon Steel \& Sumikin Engineering Co., Ltd. Japan Senior Manager, Plant \& Machinery Division, Nippon Steel \& Sumikin Engineering Co., Ltd. Japan. Iron \& Steel Division, HYL Technologies S.A. de C.V., Italy

Ironmaking DRI, Danieli \& C., Italy. 


\section{INTRODUCTION}

At 2013, world crude steel production reached more than 1,600 million tons, up by $3 \%$ compared to 2012; this is a record for the global steel production. In this contest, even if the direct reduction industry continued its firm growth with more than 74 million tons of DRI produced, crude steel production via EAF (fed with DRI or scrap) accounts only for $30 \%$ of the total, whilst $70 \%$ is still coming from Blast Furnace-Basic Oxygen Converter route. The ENERGIRON DR technology, jointly developed by Tenova HYL \& Danieli, is one of

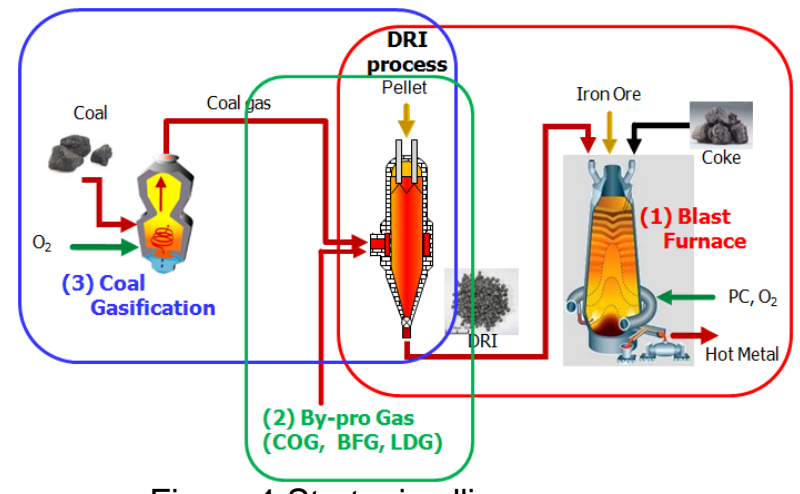

Figure 1 Strategic alliance among NSENGI, Tenova-HYL and Danieli the most viable gas-based DR technologies.

In 2013, NSENGI, Tenova HYL and Danieli have entered into an agreement to combine ENERGION DR technology into blast furnace technology and/or with syngas (by-pro gas and coal gasification) technologies.

This new alliance will allow the three companies to combine research and development activities with their respective expertise in ENERGIRON DR technology, Blast Furnace technology and Syngas technology, with the ultimate objective to develop breakthrough iron making technologies and create such EPC projects.

The objectives of the breakthrough technologies are to lower operating costs, increase productivity and/or decrease capital expenditures, also reduce $\mathrm{CO}_{2}$ emissions on integrated steelmaking facilities. The concepts of the breakthrough technologies are mainly described as follows; (Figure 1)

(1) Charging high carbon DRI (hereafter referred to as Hi-C DRI) and optimized blast furnace hardware \& operation can increase productivity and decrease hot metal cost dramatically.

(2) Beneficial using by-pro gas (COG, BFG, and LDG) in integrated steel mill can utilize reduction gas to ENERGION plant on low cost in non-natural gas producing region.

(3) Combination of coal gasification and ENERGION can expand market to non-natural gas producing region.

Above breakthrough technologies can expand the availability of ENERGIRON DR technology to the integrated steel mills and the regions where natural gas cannot be extracted.

\section{FEATURES OF ENERGIRON PROCESS}

A general process diagram is presented in Figure 2. The ENERGIRON process is designed for the conversion of iron ore (pellet or lump ore) into metallic iron, by the use of reducing gases in a solid-gas moving bed reactor. Oxygen is removed from the iron ore by chemical reactions based on hydrogen $\left(\mathrm{H}_{2}\right)$ and carbon monoxide $(\mathrm{CO})$ for the production of highly metalized DRI. [1]

Reducing gases are normally generated by self-reforming in the reduction reactor (ENERGIRON-ZR scheme). Natural gas is fed as make-up to the reducing gas circuit and oxygen is injected at the reactor inlet. The partial oxidation of natural gas with oxygen generates reducing gases in-situ $\left(\mathrm{H}_{2}\right.$ and $\left.\mathrm{CO}\right)$ and increases the operating temperature, which is required for reforming and iron ore reduction. Once in contact with the solid material inside the reactor, further reforming and cracking are carried out due to 
the catalytic effect of metallic iron. The use of oxygen allows high temperature operation and higher process efficiency.

Alternative sources of reducing gases can be used in ENERGIRON plants, such as COG, coal gasification gas, and other gases, under the same basic process scheme. Even though the chemical compositions of $\mathrm{COG}$ and natural gas are quite different, COG can be used directly in the ENERGIRON-ZR process with the same basic system configuration. Actually, as indicated in Table 1, the gas composition entering the Reactor for ENERGIRON-ZR based on COG is very similar to that of ENERGIRON-ZR based on natural gas. Remarkable advantages of this process scheme are shown as follows:

Table 1 Comparative Gas Analysis between COG and NG based Scheme in ENERGIRON-ZR

\begin{tabular}{|c|c|c|c|c|}
\hline Item & $\begin{array}{c}\text { COG make } \\
\text { up }\end{array}$ & NG make up & $\begin{array}{c}\text { COG based } \\
\text { to reduction }\end{array}$ & $\begin{array}{c}\text { NG based to } \\
\text { reduction }\end{array}$ \\
\hline $\mathrm{H}_{2}$ & $57.40 \%$ & & $50.60 \%$ & $50.00 \%$ \\
\hline $\mathrm{CO}$ & $6.50 \%$ & & $10.00 \%$ & $15.00 \%$ \\
\hline $\mathrm{CH}_{4}+\mathrm{C}_{\mathrm{m}} \mathrm{H}_{\mathrm{n}}$ & $28.80 \%$ & $97.00 \%$ & $17.50 \%$ & $25.00 \%$ \\
\hline $\mathrm{CO}_{2}$ & $2.30 \%$ & $2.50 \%$ & $1.90 \%$ & $3.00 \%$ \\
\hline $\mathrm{N}_{2}$ & $5.00 \%$ & $0.50 \%$ & $8.30 \%$ & $3.00 \%$ \\
\hline $\mathrm{H}_{2} \mathrm{O}$ & & & $9.90 \%$ & $4.00 \%$ \\
\hline
\end{tabular}

\section{(1) High carbon DRI (Hi-C DRI)}

This product typically has a carbon content of up to $5 \%$ in the form of combined carbon. Carbon in the DRI, mostly as iron carbide $\left(\mathrm{Fe}_{3} \mathrm{C}\right)$, is derived mainly from methane $\left(\mathrm{CH}_{4}\right)$ and to a lesser extent from $\mathrm{CO}$. The level of carbon is adjusted by controlling the reducing gas composition and/or oxygen injection. Most of the carbon in DRI currently being produced in the ENERGIRON ZR scheme is in the form of $\mathrm{Fe}_{3} \mathrm{C}$, as shown in Figure 3. [1]

(2) No external reducing gas reformer

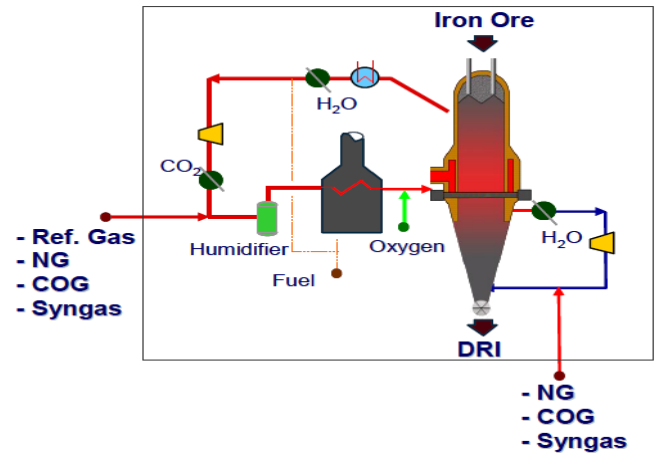

Figure 2 ENERGIRON-ZR Process [1]

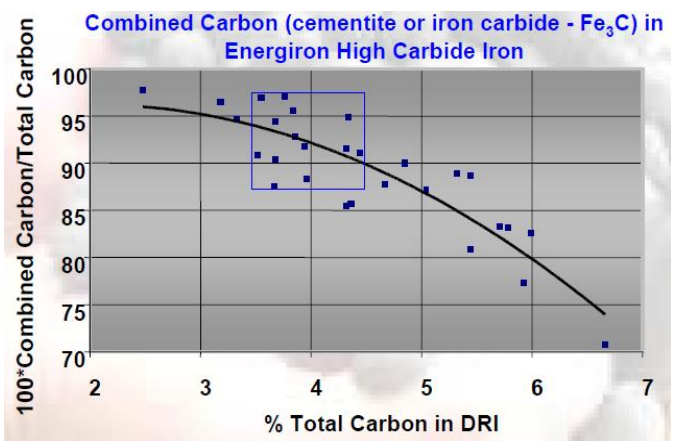

Figure 3 Combined Carbon $\left(\mathrm{Fe}_{3} \mathrm{C}\right)$ in ENERGIRON Hi-C DRI [1]

Compared to a conventional DR plant including reformer, in addition to lower operating /maintenance costs and higher DRI quality, the total investment for ENERGIRON-ZR plant is typically 10 to $15 \%$ lower, and requires only $60 \%$ of the area needed by other process plants for the same capacity. [1]

\section{CHARGING HI-C DRI AND OPTIMIZED BF OPERATION}

Many operation data of DRI/HBI charging DRI to blast furnace (BF) have indicated, hot metal production increases by around $7-8 \%$ and coke $10 \%$ of burden metallization ( $100 \mathrm{~kg}$ of metallic iron per ton of pig iron). [3]

\subsection{Additional Effects of Hi-C DRI}

Moreover, in case of $\mathrm{Hi}-\mathrm{C}$ DRI to be produced by ENERGIRON-ZR, there are additional effects as follows:

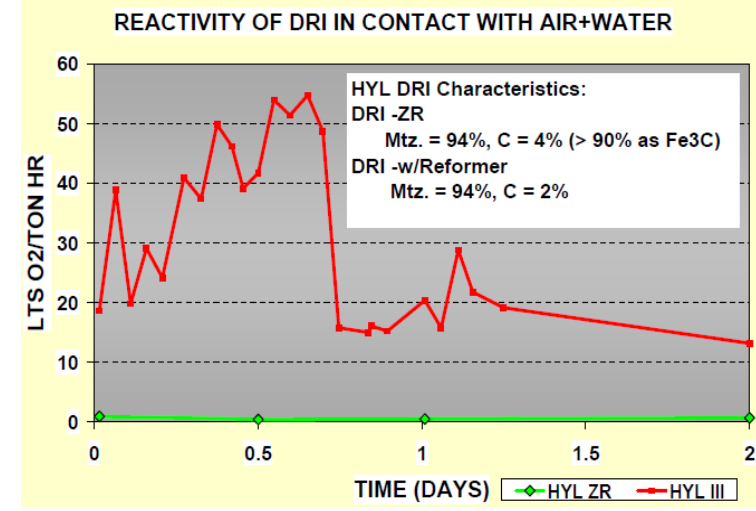

Figure 4 Reactivity of DRI in contact with air and water 


\section{(1) No hot briquetting}

High carbon $\left(\mathrm{Fe}_{3} \mathrm{C}\right) \mathrm{DRI}$ is characterized by a higher stability in transportation and storage, as shown in Figure 4. Therefore, it can be stored in the yard and charged to BF as shape as DRI, it is easy to handle by conveyers and hoppers of BF, and easy to be reduced and melted in $\mathrm{BF}$, because of high porosity, compared to low carbon $\mathrm{HBI}$.

\section{(2) Improvement of reducing in BF}

Wustite (FeO) in $\mathrm{Hi}-\mathrm{C}$ DRI can be reduced by carbon in $\mathrm{Hi}-\mathrm{C}$ DRI by only heated. And generated $\mathrm{CO}$ gas from $\mathrm{Hi}-\mathrm{C} \mathrm{DRI}$ can advance to reduce the iron ore around $\mathrm{Hi}-\mathrm{C} \mathrm{DRI}$, compared to low carbon $\mathrm{HBI}$, as shown in Figure 5.

\section{(3) Improvement of permeability in BF}

Metallic $\mathrm{Fe}$ does not produce low-melting-point material with slag. Because of charging $\mathrm{Hi}-\mathrm{C} \mathrm{DRI}$ to $\mathrm{BF}$, metallic $\mathrm{Fe} \%$ is increased above cohesive zone in $\mathrm{BF}$, therefore the gas permeability in BF is improved, as shown in Figure 6.
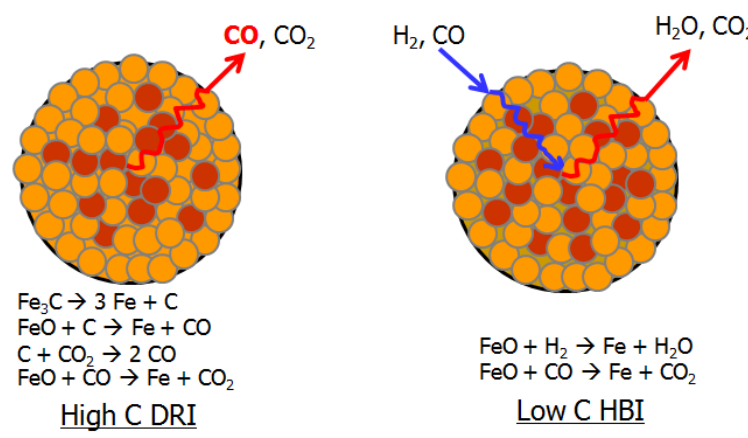

Figure 5 Improvement of reducing in BF by Hi-C DRI

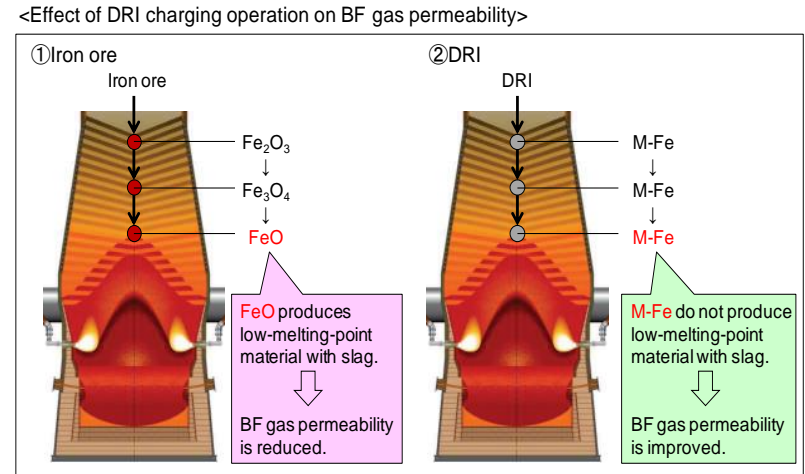

Figure 6 Improvement of permeability in BF by $\mathrm{Hi}-\mathrm{C}$ DRI

\subsection{Additional Effects of Optimum Hardware and Operation of BF}

\subsubsection{Control gas flow uniform}

Figures 7 and 8 show the gas flow under stable and unstable BF operations respectively. The point for achieving lower coke rate and higher productivity operation is the control gas flow uniform when DRI are charged to BF.

To make the gas flow uniform, it is necessary following two points.

i) Make a uniform burden distribution in radius direction in the furnace

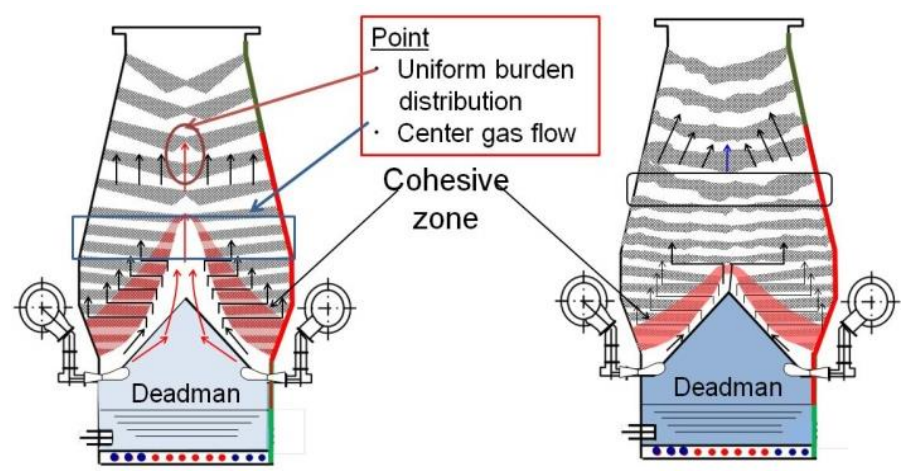

Figure 7 Ideal gas flow

Figure 8 Non-ideal gas flow

ii) Provide a proper center gas flow

Figure 7 shows an ideal furnace inner condition. The burden is distributed uniformly circumferentially and a center gas flow is provided. As a result, gas flow becomes uniform and an in-furnace reaction progresses efficiently, enabling low coke rate operations to be accomplished. In contrast, in Figure 8 the burden is not uniform distribution and no center gas flow. Thus, gas flow is non-uniform, causing instable operation, which results in an increased coke rate. [4]

\section{(1) Technology for making gas flow uniform}

Since 1974, NSENGI has been delivered more than 80 units of top charging equipment to Nippon Steel \& Sumitomo Metal Gr. and other steel companies in the world, and has 
also been continuously making improvements to make in-furnace gas flow uniform circumferentially and provide a center gas flow.

Figure 9 shows the flow of materials from the receiving hoppers to chutes and then to the furnace inside of the conventional top charging equipment. As shown in Figure 9-(A), materials are charged into the furnace lopsidedly, because there is generally the horizontal component of the velocity in parallel hoppers. However, NSENGI has the technology of preventing inertia-caused uneven flows from being created and of charging materials into the furnace uniformly circumferentially as shown in Figure 9-(B).

In addition, for the particle sizes of the materials to be discharged from the hoppers, fine particles are discharged first and coarse particles are fed later. As shown in Figure 10-(A), an occurrence of time-series size segregation of the material causes finer particles to be apt to be concentrated at the furnace center, making a center gas flow weaker. NSENGI has the technology of controlling material flows so that the distribution of the particle size is constant in time series at the hopper outlets as shown in Figure 10-(B). Therefore, size segregation in the circumferentially direction in the furnace is eliminated, enabling the gas flow to be distributed as shown in Figure 7. [4]

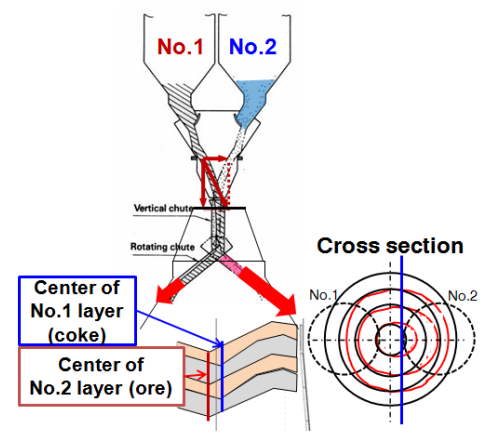

(A)Non ideal material distribution

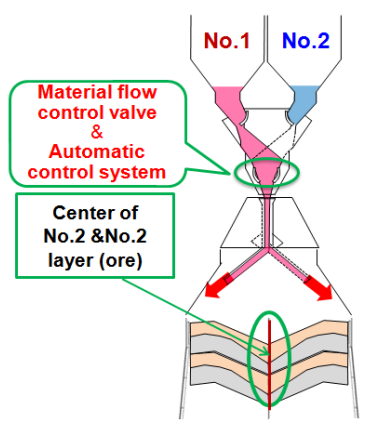

(B) Ideal material distribution

Figure 9 Change in in-furnace material distribution by improvement of top charging equipment

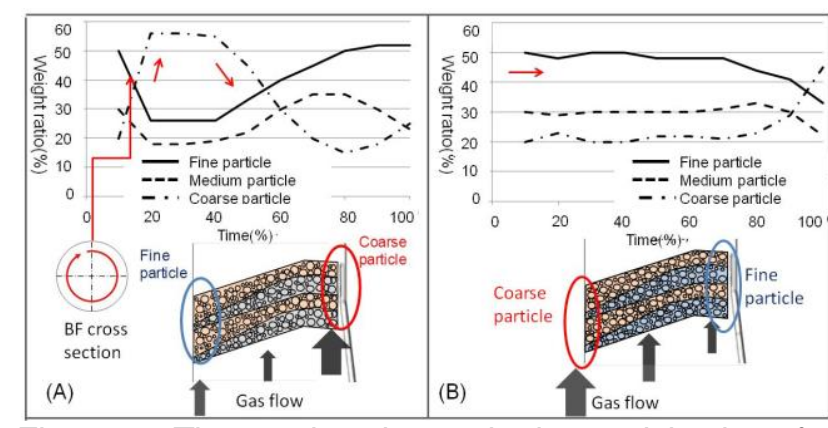

Figure 10 Time-series change in the particle size of materials discharged from hoppers and their in-furnace distribution

\section{(2) Advanced top charging equipment}

To make gas flow further uniform and provide a center gas flow, NSENGI proposes the advanced top charging equipment based on experiences and technologies attained from a lot of delivery records.

As shown in Figure 11, in the advanced top charging equipment, the rotating speed of the chute has improved to $12 \mathrm{rpm}, 1.5$ times that of conventional type due to weight reduction of the rotating section such as the chutes and a reduced moment of inertia. Moreover, the tilting speed becomes three times faster, providing a very high response. Therefore, the advanced top charging

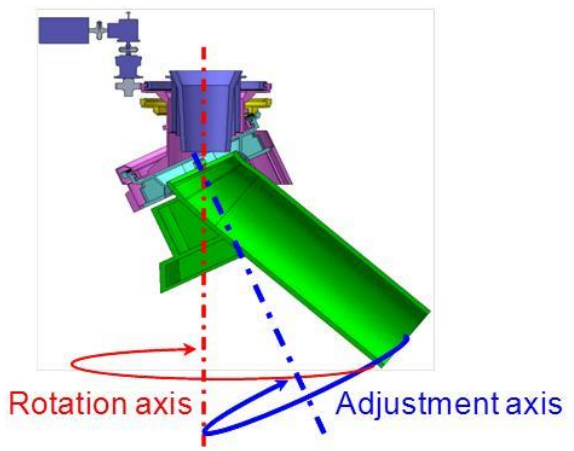

Figure 11 Advanced top charging equipment equipment can support a variety of material distribution methods. Furthermore, the chute contour was improved, so that the material does not disperse, improving the distribution accuracy.

As a result, further uniform gas flow and the formation of a center gas flow are accomplished.

In addition, the conventional type required more than 11 gears to perform tilting, but the advanced top charging equipment has only three gears by simplifying the mechanics. The simplification of the construction enables the material falling height to be lower by 1 
$\mathrm{m}$. This reduces the pulverization of the material due to crash, preventing draft from getting worse.

\subsubsection{Optimum operation of BF}

Basically, when coke ratio is decreased by other fuel injection such as $\mathrm{PCl}$, the gas permeability resistance in $\mathrm{BF}$ is increased ( $\mathrm{K}$ value is increased), so decreasing coke ratio is limited by limit of $K$ value of $B F$ as shown in Figure 12.

However, the gas permeability resistance in $\mathrm{BF}$ ( $\mathrm{K}$ value) can be decreased by i) Charging $\mathrm{Hi}-\mathrm{C}$ DRI, ii) Uniform gas flow by optimum material charging and iii) Optimizing other operation parameters as shown in Figure 12.

The BF operation parameters can be optimized to achieve higher productivity and lower coke ratio, by the operation simulation model of BF.

As a result, around double to the conventional effects has been verified to the BF productivity increase and coke ratio reduction.

1) Hot metal cost down merit is estimated 6 to 10 US\$ per ton-steel.

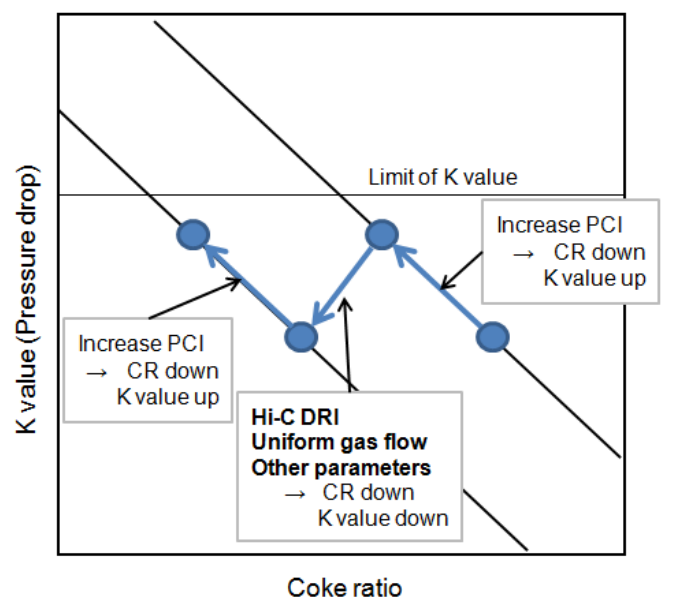

Figure 12 Relation between coke ratio and Permeability

2) The reinforcing coke oven and BF is not necessary for increasing steel production, because productivity is increased and coke rate is decreased by charging $\mathrm{Hi}-\mathrm{C}$ DRI to BF.

3) Old small BFs can be shut down, by charging Hi-C DRI to new big BFs and increasing productivity.

4) Life time of coke ovens can be prolonged, or old small coke ovens can be shut down, by charging $\mathrm{Hi}-\mathrm{C} \mathrm{DRI}$ to $\mathrm{BF}$ and decreasing coke rate.

\section{BENEFICIAL USING BY-PRO GAS (COG, BFG, LDG) IN INTEGRATED STEEL MILL}

\subsection{Gas balance and feeding to ENERGIRON in integrated steel mill}

Beneficial using by-pro gas (COG, BFG, and LDG) in integrated steel mill can utilize reduction gas to ENERGION plant on low cost in non-natural gas producing region.

Figure 13 and 14 show the generation and usage of by-pro gas (COG, BFG and LDG) in the average integrated steel mill. By-pro gas is generated approx. $2.2 \mathrm{Gcal} / \mathrm{t}-\mathrm{steel}$, and approx. $40 \%$ (approx. $0.9 \mathrm{Gcal} / \mathrm{t}-\mathrm{s}$ ) of it is used for power generation.

If DRI is produced by ENERGIRON using by-pro gas and charged to BF $100 \mathrm{~kg} / \mathrm{t}-\mathrm{s}$, bypro gas is consumed approx. $0.25 \mathrm{Gcal} / \mathrm{t}-\mathrm{s}$, corresponding approximately to $30 \%$ of bypro gas for power generation.

In case of power generation efficiency is $3 \mathrm{Gcal} / \mathrm{MWh}$, by-pro gas cost is $20 \mathrm{US} \$ / \mathrm{Gcal}$ and purchased power price is 80US\$/MWh, the benefit of power generation is 10 US $\$$ /MWh, corresponding to 0.8 US $\$$ per ton-steel as shown in Figure 15.

On the other hand, benefit of DRI producing and charging to BF is 6 to 10 US\$ per tonsteel, it is much bigger than one of power generation. Therefore, approx. $30 \%$ of by-pro gas which is used for power generation can be converted to producing DRI, considering gas balance and benefit. 


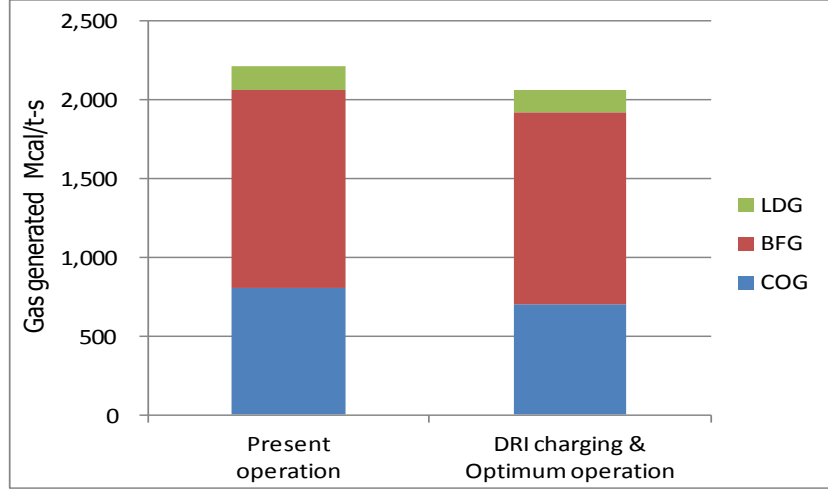

Figure 13 Example of by-pro gas generated in intearated steel mill

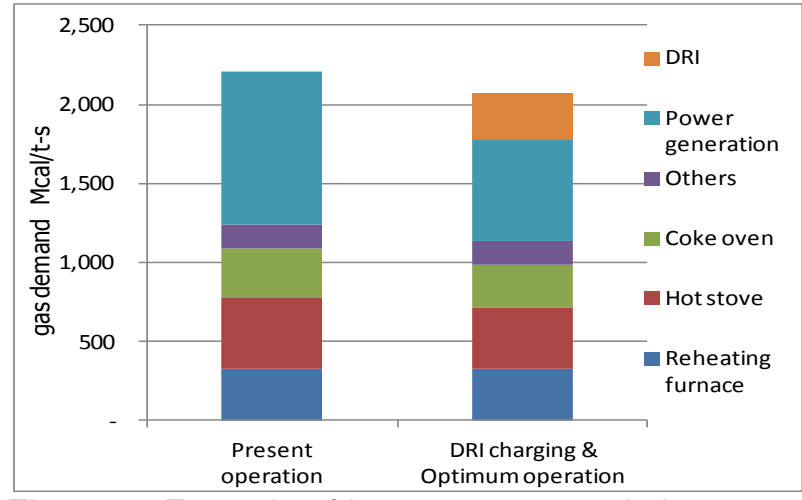

Figure 14 Example of by-pro gas usage in integrated steel mill

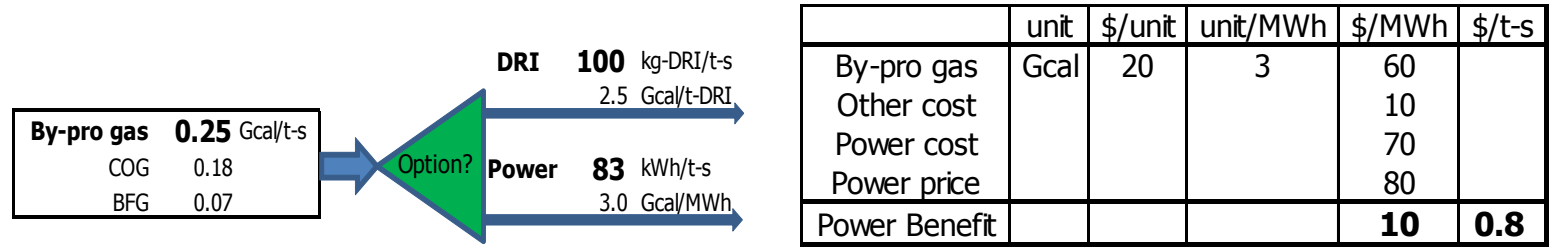

Figure 15 Comparison of benefit by DRI produce and power generation

\subsection{Improvement of COG balance by applying NSEGl's energy saving system}

However, in the cases of lower COG generation because of purchasing coke or using low volatile coal, or higher COG consumption because of lower heat efficiency of facilities such as reheating furnaces, COG might run short by around $0.18 \mathrm{Gcal} / \mathrm{t}$-s for utilizing to ENERGIORN plant. If power network is too week and purchased power cannot be increased, the power might run short by around $83 \mathrm{kWh} / \mathrm{t}-\mathrm{s}$ for utilizing by-pro gas to ENERGIORN plant as calculated in Figure 15.

In these cases, if the energy saving facilities of NSENGI are applied, enough COG can be fed to ENERGIRON plant, and enough power can be generated as shown in Table 2 and 3 .

Table 2 Example of COG balance improvement

\begin{tabular}{|c|c|}
\hline COG demand & Gcal/t-s \\
\hline Increase for ENERGIRON & +0.18 \\
\hline (1)Decrease by NSENGI HRS & $-0.09 \sim-0.19$ \\
\hline (2)Decrease by Regenerative burner & -0.1 \\
\hline COG demand decrease total & $-0.01 \sim-0.11$ \\
\hline
\end{tabular}

Table 3 Example of Power balance improvement

\begin{tabular}{|c|c|c|}
\hline \multicolumn{1}{|c|}{ Power generation } & $\mathrm{kWh} / \mathrm{t}-\mathrm{S}$ & Remarks \\
\hline Decrease by ENERGIRON & -83 & $\mathrm{COG}+\mathrm{BFG}=+0.25 \mathrm{Gca} / \mathrm{t}$ \\
\hline (1)Increase by NSENGI HRS & +20 & $\mathrm{COG}+\mathrm{BFG}=-0.06 \mathrm{Gca} / \mathrm{t}$ \\
\hline (2)Increase by Regenerative burner & +33 & $-0.1 \mathrm{Gcal} / \mathrm{t}$ \\
\hline (3)Increase by MVEP & $+14 \sim+54$ & $\begin{array}{c}\text { Wet type } \rightarrow \mathrm{MVEP}:+14 \\
\text { MVEP }+\mathrm{TRT}:+54\end{array}$ \\
\hline Power generation increase total & $-16 \sim+24$ & \\
\hline
\end{tabular}

(1) Hot stove waste gas heat recovery system (NSENGI HRS)

Fuel gas used in a hot stove is mainly BFG with additional high calorie gas such as COG. For example, high calorie gas consumption is $0.1-0.17 \mathrm{Gcal} / \mathrm{t}-\mathrm{s}$, to achieve high dome temperature. And, the combustion waste gas of hot stove has high temperatures as approx. $300^{\circ} \mathrm{C}$ and high thermal energy, but is discharged into the air through the stack. Recovering this discharged energy to preheat the fuel gas and combustion air allows the fuel gas and combustion air, which contribute to decrease high calorie gas consumption, therefore running cost of hot stove is reduced.

Figure 16 shows the gas flow of NSENGI's Waste gas Heat Recovery System with heat medium (hereafter referred to as NSENGI HRS). NSENGI HRS consists of a heat 
exchanger for receiving heat from the waste gas ("HE-1" in Figure 16), heat exchangers for heating the fuel gas and combustion air ("HE-2" and 3 in Figure 16), and a temperature control value that controls heat medium temperature ("XV-1" in Figure 16). It transfers heat recovered by the heat medium oil to preheat the fuel gas and combustion air.

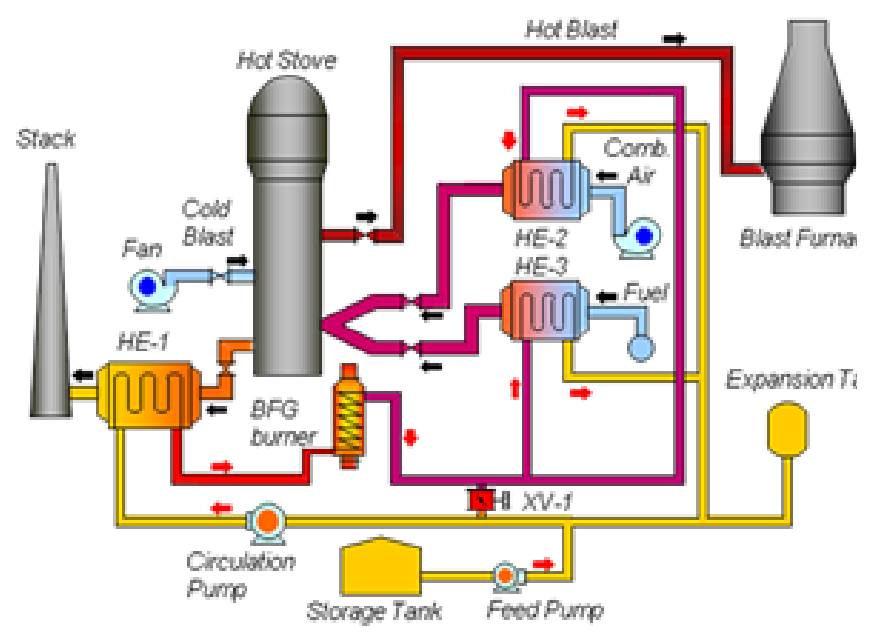

Fiaure 16 Gas flow of NSENGI HRS

Additionally, by preheating BFG and combustion air further, it is possible to achieve the specified dome temperature only with BFG. To realize a solely BFG operation, BFG and combustion air are preheated further by use of "BFG burner" in Figure 16. In the operation by NSENGI HRS, the heat medium is heated by the waste gas of "BFG burner" directly, and the heat medium heats BFG and combustion air. [4]

As the result, COG consumption can be decreased by $0.1-0.17 \mathrm{Gcal} / \mathrm{t}-\mathrm{s}$

\section{(2) Regenerative burner for re-hearting furnace}

In the average integrated steel mill, bypro gas, such as COG and/or LDG, are consumed around 0.35 to $0.4 \mathrm{Gcal} / \mathrm{t}-\mathrm{s}$ for the reheating furnaces for slab, bloom and/or billet. Figure 17 shows the operating principle of regenerative burner system. NSENGI have constructed 16 reheating furnaces applied regenerative burner system, and supplied 130 pairs of original regenerative burner.

In case of conventional burner, the combustion air is preheated by high temperature exhaust gas using the metal type recuperators to around $400^{\circ} \mathrm{C}$, and

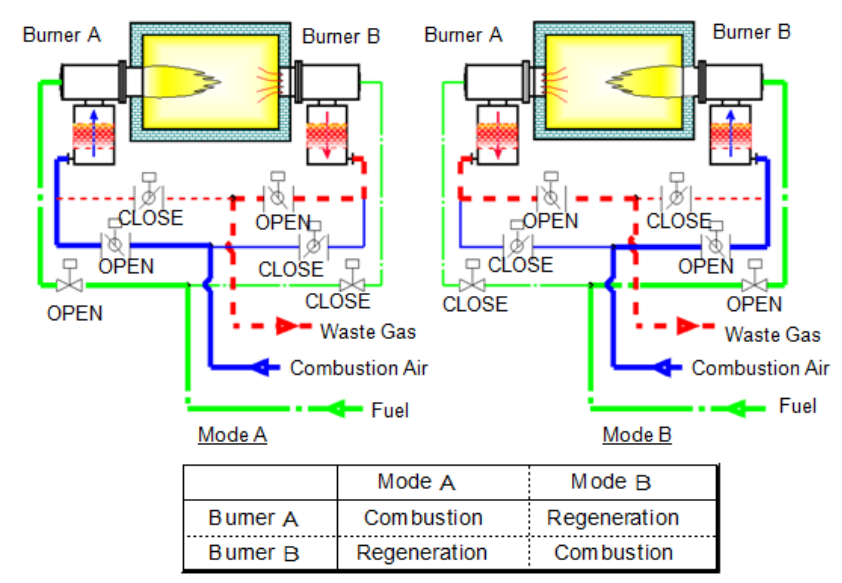

Figure 17 Operating principle of regenerative burner system heat recovery efficiency is approx. $30 \%$.

On the other hand, in the regenerative burner type, a burner with a regenerative medium is placed in both sidewalls of the reheating furnace. The twin burner in both sidewalls burns in turn, Induced Draft Fan located in the flue absorb approximately $80 \%$ of combustion waste gas in a high temperature state, in a regenerative medium located just after a burner, the high temperature exhaust gas does heat exchange to combustion air. Therefore, we can get preheating air about $1,100^{\circ} \mathrm{C}$, exhaust heat recovery efficiency largely improves to approx. $70 \%$. [5] 
As the result, gas consumption can be decreased by approx. 30\%, corresponding approximately to $0.1 \mathrm{Gcal} / \mathrm{t}-\mathrm{s}$

\section{(3) Dry-type Multi-Vessel Electrostatic Precipitator}

Figure 18 shows the gas flow of NSENGl's Multi-Vessel Electrostatic Precipitator (hereafter referred to as MVEP). [4] By the top-gas pressure recovery turbine (hereafter referred to as TRT) adoption the downstream of this equipment, the pressure and temperature energy of top gas is recovered as electric power. As a result, it is required to minimize the drops in gas pressure and

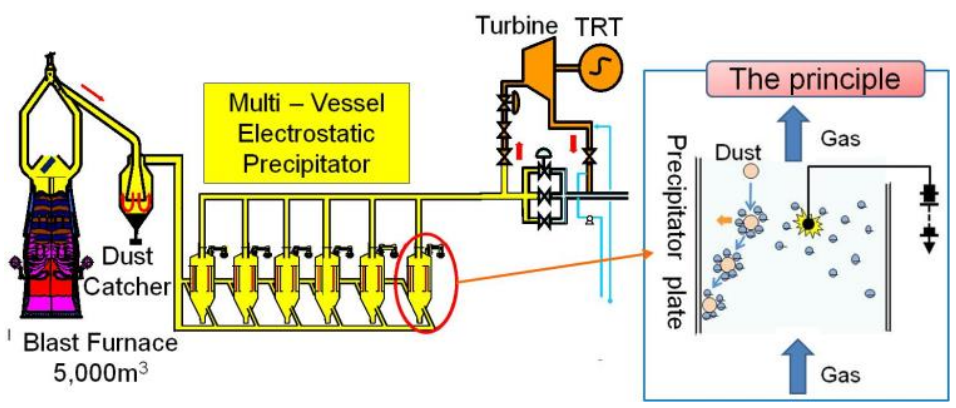

Figure 18 Dry-type Multi-Vessel Electrostatic Precipitator (MVEP) temperature during gas cleaning from the viewpoint of energy saving.

In case of MVEP, the inlet temperature of TRT is increased by approx. $80^{\circ} \mathrm{C}$, and the inlet pressure of TRT is increased by approx. $3600 \mathrm{mmAq}$, compared to conventional wet type. As the result, power recovery is increased by approx. $30 \%$, to $54 \mathrm{kWh} / \mathrm{t}-\mathrm{p}$ from $40 \mathrm{kWh} / \mathrm{t}-$ p, compared to conventional wet type.

\section{COMBINATION OF COAL GASIFICATION AND ENERGION}

The Combination of coal gasification process and ENERGION process can be propounded, in default of natural gas and by-pro gas supply, such as the mini-mill (DRI + EAF) in non-natural gas producing region.

As explained chapter 2, ENERGION process has a wide acceptable range for reduction gas, therefore, every type of coal gasification, such as entrained flow type, fluidized type and fixed bed type can be applied with ENERGION process.

\section{(1) ECOPRO ${ }^{* * 1)}$}

NSENGI have developed the innovative coal gasification technology using the high efficient two-stage entrained flow gasifier named ECOPRO, and testing has been completed at the pilot plant with a coal feed of 20 ton/day from 2003 to 2009 , supported by Japanese Government (METI) through Japan Coal Energy Center (JCOAL), and verified as follows, [6]

1) World highest energy efficiency of $85 \%$,

5 to $10 \%$ higher than the conventional gasifier

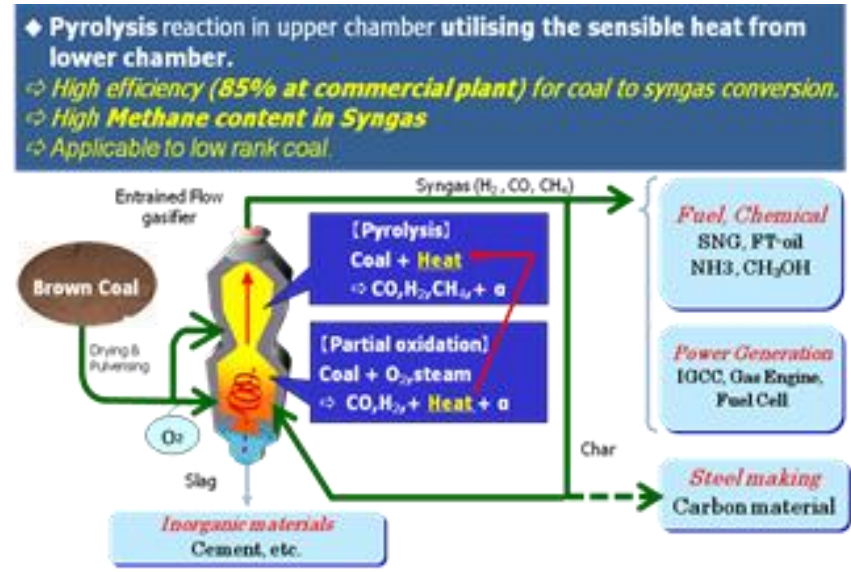

Figure 19 Characteristics of ECOPRO

2) Applicability of the process to low-rank coals including brown coal and operating stability NSENGI plan to install and demonstrate the middle size plant (200 ton/day) in China, and will commercialize the large scale plant (more than 1000 ton/day) in a couple of years. By application of ECOPRO and ENERGIRON, total energy efficiency will be increased by 5 $10 \%$. ( $\left.{ }^{*} 1\right)$ Stands for Efficient Co-Production with Coal Flash Partial Hydro-pyrolysis Technology 


\section{(2) ESCAP $\left.{ }^{*} 2\right)$}

The $\mathrm{CO}_{2}$ generation is increased by application with coal gasification, compared to natural gas, however, the $\mathrm{CO}_{2}$ emission to atmosphere is not increased so much, because of $\mathrm{CO}_{2}$ selective removal in the case of ENERGIRON process. In the case of application with coal gasification, reducing reaction energy of $\mathrm{CO}_{2}$ becomes more important, considering total energy efficiency.

NSENGI have developed the most efficient energy cut-off chemical absorption process for $\mathrm{CO}_{2}$ capture, named ESCAP in the project sponsored by NEDO (New Energy and Industrial Technology Development Organization) in Japan, as shown in Figure 20.

Achievements of new absorbent, which had been developed by NSSMC (Nippon Steel \& Sumitomo Metal Corporation) and RITE (Research Institute of innovative Technology for Earth), were $42 \%$ reduction in reaction energy $\left(2.3 \mathrm{GJ} / \mathrm{t}-\mathrm{CO}_{2}\right)$ as shown in Figure 21 , and $30 \%$ increase in $\mathrm{CO}_{2}$ absorption relative to conventional MEA (Mono Ethanol Amine).

The commercial plant of ESCAP with $120 \mathrm{t}-\mathrm{CO}_{2} /$ day was constructed in Muroran Works of NSSMC in September 2014, and have been operated well on planed condition. The recovered $\mathrm{CO}_{2}$ from the hot stove exhaust gas of $\mathrm{BF}$ is used mainly dry ice and beverage in Hokkaido area. [7] $\quad\left({ }^{*} 2\right)$ Stands for Energy Saving $\mathrm{CO}_{2}$ Absorption Process

\section{CONCLUSION}

In 2013, NSENGI, Tenova HYL and Danieli \& C have entered into an agreement to combine ENERGION DR technology into blast furnace technology and/or with syngas technologies.

The outlines of the breakthrough technologies are mainly described as follows;

(1) Charging Hi-C DRI and optimized BF hardware \& operation can increase productivity and decrease hot metal cost dramatically, because of improving reduction, permeability and flow control in the BF. Hot metal cost down merit is estimated 6 to $10 \mathrm{US} \$$ /ton-steel. The reinforcing coke oven and BF is not necessary for increasing steel production, because productivity is increased and coke rate is decreased by charging $\mathrm{Hi}-\mathrm{C} \mathrm{DRI}$ to $\mathrm{BF}$.

Old small BF and coke oven can be shut down, by charging Hi-C DRI to new big BF and increasing productivity.

(2) By-pro gas can be converted from power generation to producing Hi-C DRI, considering gas balance and benefit.

Even if there is shortage of COG, COG can be fed to ENERGIRON plant, by application of the energy saving facilities of NSENGl.

(3) Combination with coal gasification can expand market to non-natural gas producing region. Total efficiency can be improved more by application of ECOPRO (high efficiency coal gasifier) and ESCAP (Energy Saving $\mathrm{CO}_{2}$ Absorption Process).

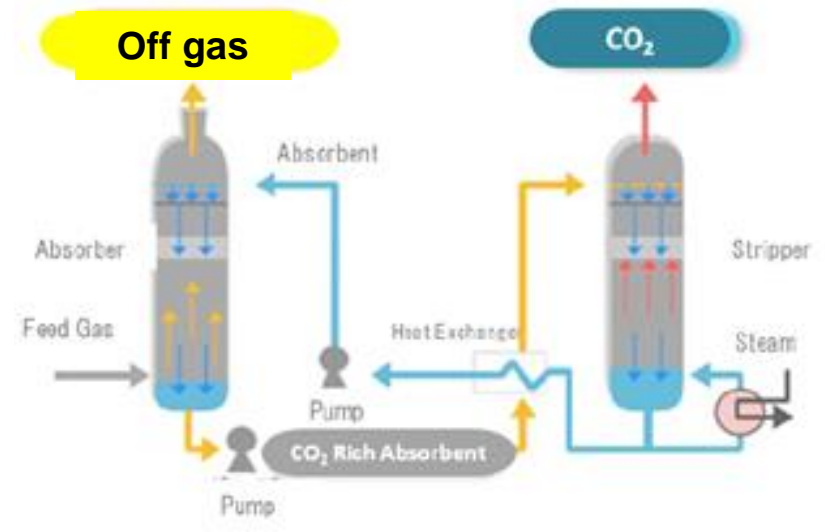

Figure 20 ESCAP
Reaction energy $\left(\mathrm{GJ} / \mathrm{t}-\mathrm{CO}_{2}\right)$

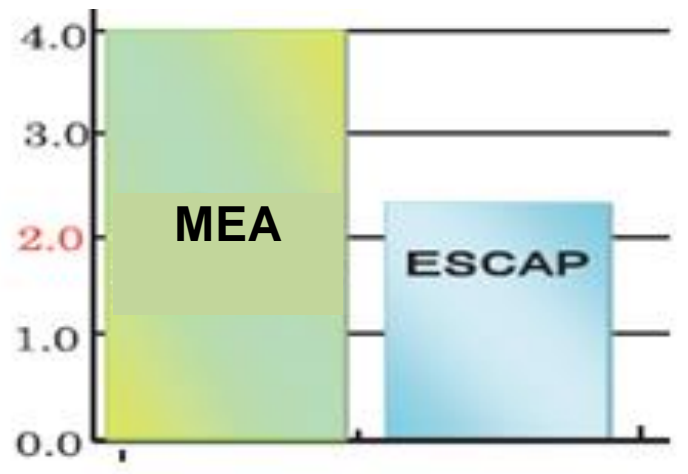

Figure 21 Reaction energy of ESCAP 
NSENGI, Tenova HYL and Danieli will further contribute to lower operating costs, increase productivity and/or decrease capital expenditures, also reduce $\mathrm{CO}_{2}$ emissions on integrated steelmaking facilities through spread of our technologies.

\section{REFERENCES}

1 Becerra, J.; Duarte, P.: HYL ENERGIRON - The Most Economical Way to High Quality DRI; Arab Iron \& Steel Union Conference 2007, Muscat, Oman, March 2007

2 Becerra, J.; Martinis, A.: Alternative Energy Sources, $\mathrm{CO}_{2}$ Recovery Technology and Clean Environment Compliance - Integral Compositions of Energiron Technology; January 2008, Iron \& Steel Review

3 Home page of International Iron Metallics Association (IIMA)

4 Usui, K.; Maesaka, N.; Morimitsu, K.; Ishikawa, H.; Tomisaki, S.: Energy-saving Technologies for Blast Furnaces Realized by Nippon Steel \& Sumikin Engineering; The 44th Ironmaking and Raw Material Seminar, September 15th to 18th, 2014, Belo Horizonte, MG, Brazil.

5 Kubo, K: Replacement of a Reheating Furnace of a Wide Flange Mill; South East Asia Iron and Steel Institute (SEAISI) 2014, Kuala Lumpur, Malaysia

6 Takeda, S.; Kosuge, K.; Itonaga, M.; Kato, K.: Development of ECOPRO coal gasification process - High efficiency coal gasification technology applicable to low rank coals; Nippon Steel Sumikin Engineering Co., Ltd. Technical Review, Vol.6, 2015

7 Mimura, T.; Igarashi, M.; Tezuka, M.: High Performance $\mathrm{CO}_{2}$ Recovery Process; ADIPEC 2013 Technical Conference, 10-13th November 